\title{
Cellular Response to Ciprofloxacin in Low-Level Quinolone-Resistant Escherichia coli
}

\begin{abstract}
Jesús Machuca ${ }^{1,2}$, Esther Recacha ${ }^{1,2}$, Alejandra Briales ${ }^{3,4}$, Paula Díaz-de-Alba', Jesús Blazquez ${ }^{2,3,5}$, Álvaro Pascual ${ }^{1,2,3}$ and José-Manuel Rodríguez-Martínez ${ }^{2,3,4 *}$

1 Unidad Intercentros de Enfermedades Infecciosas, Microbiología y Medicina Preventiva, Hospital Universitario Virgen Macarena y Virgen del Rocío, Seville, Spain, ${ }^{2}$ Instituto de Biomedicina de Sevilla, Hospital Universitario Virgen del Rocio/Consejo Superior de Investigaciones Científicas/Universidad de Sevilla, Sevilla, Spain, ${ }^{3}$ Red Española de Investigación en Patología Infecciosa, Instituto de Salud Carlos III, Madrid, Spain, ${ }^{4}$ Departamento de Microbiología, Universidad de Sevilla, Sevilla, Spain, ${ }^{5}$ Centro Nacional de Biotecnología, Consejo Superior de Investigaciones Científicas, Madrid, Spain
\end{abstract}

Bactericidal activity of quinolones has been related to a combination of DNA fragmentation, reactive oxygen species (ROS) production and programmed cell death (PCD) systems. The underlying molecular systems responsible for reducing bactericidal effect during antimicrobial therapy in low-level quinolone resistance (LLQR) phenotypes need to be clarified. To do this and also define possible new antimicrobial targets, the transcriptome profile of isogenic Escherichia coli harboring quinolone resistance mechanisms in the presence of a clinical relevant concentration of ciprofloxacin was evaluated. A marked differential response to ciprofloxacin of either up- or downregulation was observed in LLQR strains. Multiple genes implicated in ROS modulation (related to the TCA cycle, aerobic respiration and detoxification systems) were upregulated (sdhC up to 63.5-fold) in mutants with LLQR. SOS system components were downregulated (recA up to 30.7-fold). yihE, a protective kinase coding for PCD, was also upregulated (up to 5.2-fold). SdhC inhibition sensitized LLQR phenotypes (up to $\Delta$ Log $=2.3$ after $24 \mathrm{~h}$ ). At clinically relevant concentrations of ciprofloxacin, gene expression patterns in critical systems to bacterial survival and mutant development were significantly modified in LLQR phenotypes. Chemical inhibition of SdhC (succinate dehydrogenase) validated modulation of ROS as an interesting target for bacterial sensitization.

Keywords: ciprofloxacin, low-level quinolone resistance, survival, transcriptomic, global response, sensitization

\section{INTRODUCTION}

Enterobacteriaceae, particularly Escherichia coli, are among the most common organisms causing community, nosocomial, and opportunistic infections (Vila et al., 2016). Fluoroquinolones are commonly used for empirical and directed therapy in infections caused by E. coli (Vila et al., 2016). Over the past three decades, quinolone resistance in Enterobacteriaceae from human and veterinary isolates has increased (Rodríguez-Martínez et al., 2016b). Known mechanisms of fluoroquinolone resistance occur principally through chromosomal mutations in genes encoding the quinolone targets (DNA gyrase and topoisomerase IV), and to a lesser extent through decreased permeability (implicating upregulation of the AcrAB-TolC multidrug efflux pump) (Blair et al., 2015; Hooper and Jacoby, 2015). Additionally, plasmid-mediated quinolone resistance (PMQR) 
mechanisms (Qnr proteins that protect the quinolone targets; the acetylation of ciprofloxacin and norfloxacin by $\mathrm{Aac}\left(6^{\prime}\right)-\mathrm{Ib}-\mathrm{cr}$; and the plasmid-mediated efflux pumps, QepA and OqxAB) have also been described and are epidemiologically relevant (Rodríguez-Martínez et al., 2011, 2016b; Jacoby et al., 2014). All these determinants (chromosomal or plasmid mediated) on their own confer low-level quinolone resistance (LLQR), and multiple mechanisms must be combined to achieve clinical levels of resistance (Morgan-Linnell and Zechiedrich, 2007; MorganLinnell et al., 2009; Briales et al., 2011; Machuca et al., 2014).

It is of crucial medical importance to understand the molecular mechanisms that promote the development of antimicrobial resistance as well as to clarify the molecular responses to antimicrobials that lead to the survival or death of the cell (Palmer and Kishony, 2013; Baym et al., 2016). The bactericidal activity of quinolones in bacteria has been related to a combination of DNA fragmentation, reactive oxygen species (ROS) production and programmed cell death (PCD) systems mediated by toxin-antitoxin (TA) modules such as mazEF (Drlica et al., 2009; Erental et al., 2014; Zhao et al., 2015). The SOS system response has also been postulated as a formidable strategy against aggressions such as antimicrobial exposure, inducing the transient expression of mutational machinery (Baharoglu and Mazel, 2014). The intensity and role of all these processes implicated in survival and mutant emergence would be proportional to drug concentration (and a function of susceptibility levels directly related to MIC values).

The impact of LLQR mechanisms on the decreased bactericidal effect of these antimicrobial agents and their increased influence on the emergence of high-level resistance seems to be clear. However, the underlying molecular systems responsible for this phenomenon in clinical setting still need to be clarified. In order to do this in terms of genetic expression, this study evaluated the transcriptome profiling of isogenic E. coli harboring frequent quinolone resistance mechanisms (chromosomal and plasmid-mediated) in the presence of clinically significant concentrations of ciprofloxacin (breakpoint for reduced susceptibility which is near to $1 / 2$ of serum Cmax) (Mandell et al., 2010). We also evaluated enhancing ciprofloxacin activity by targeting ROS modulation, which was greatly altered in LLQR strains.

\section{MATERIALS AND METHODS}

\section{Bacterial Strains}

Escherichia coli ATCC $^{\circledR} 25922^{\mathrm{TM}}$ was used as the background strain. This microorganism is a recommended CLSI control strain used worldwide for antimicrobial susceptibility testing (including quinolones). First, four LLQR isogenic strains were tested using a global transcriptomic approach: ATCC 25922 (wild-type, MIC for ciprofloxacin $0.004 \mu \mathrm{g} / \mathrm{mL}$ ); EC14 (E. coli ATCC 25922 coding for QnrS1, MIC for ciprofloxacin $0.125 \mu \mathrm{g} / \mathrm{mL}$ ); EC19 (E. coli ATCC 25922 with deleted marR gene and coding for QnrS1, MIC for ciprofloxacin $0.5 \mu \mathrm{g} / \mathrm{mL}$ ); and EC24 (E. coli ATCC 25922 with the Ser83Leu substitution in GyrA and coding for QnrS1, MIC for ciprofloxacin $1 \mu \mathrm{g} / \mathrm{mL}$ )
(Table 1; Machuca et al., 2014). All of these were susceptible to quinolones according to CLSI breakpoints (CLSI). Second, E. coli ATCC 25922 and two isogenic mutants harboring chromosomal and/or PMQR mechanisms (EC04: E. coli ATCC 25922 with the Ser83Leu substitutions in GyrA and Ser80Arg in ParC, MIC for ciprofloxacin $0.5 \mu \mathrm{g} / \mathrm{mL}$; and EC59: E. coli ATCC 25922 with the Ser83Leu and Asp87Arg substitutions in GyrA and Ser80Arg in ParC, deleted marR gene and coding for QnrS1, MIC for ciprofloxacin $32 \mu \mathrm{g} / \mathrm{mL}$ ) were used for $s d h C$ (succinate dehydrogenase complex subunit $\mathrm{C}$ ) or $c y o A$ (cytochrome oxidase subunit II) inactivation, as described (Table 1; Datsenko and Wanner, 2000; Machuca et al., 2014). qnrS1 gene was cloned into pBK-CMV vector as described previously (Machuca et al., 2014). Empty pBK-CMV vector was used as control and also introduced into E. coli ATCC 25922 and EC04 for comparison.

\section{Experimental Conditions and Microarray RNA Analysis}

Escherichia coli ATCC 25922 (wild-type) and isogenic EC14, EC19, EC24 (LLQR) strains were tested to evaluate the global response to relevant fixed concentrations of ciprofloxacin ( $1 \mu \mathrm{g} / \mathrm{mL}$, the breakpoint for reduced susceptibility according to CLSI and near to $1 / 2$ of serum Cmax) (Mandell et al., 2010). Transcriptomic profile of E. coli ATCC 25922 was also compared to EC14 in absence of ciprofloxacin as control. All were susceptible to quinolones according to CLSI breakpoints (CLSI). Cultures were started from single colonies and grown overnight in $25 \mathrm{ml}$ of LB (Luria Bertani medium, Becton Dickinson, Le Pont-de-Claix, France). These cells were diluted 1:100 and grown to cell concentrations of $4 \times 10^{8}$ cells $/ \mathrm{ml}$ $\left(\mathrm{OD}_{600 \mathrm{~nm}}=0.4\right.$, exponential phase $)$ for treatment. Three biological replicates per genotype were incubated at $1 \mu \mathrm{g} / \mathrm{mL}$ ciprofloxacin for $60 \mathrm{~min}$ (i.e., 250xMIC for E. coli ATCC 25922, 8xMIC for EC14, 2xMIC for EC19 and 1xMIC for EC24). Approximately $10^{9}$ cells $(2 \mathrm{ml})$ were taken for RNA isolation. The pellet was processed immediately. The pellet was pre-incubated in RNAprotect Bacteria Reagent (Qiagen, Hilden, Germany). RNA extraction was performed using the RNeasy Mini Kit (Qiagen, Hilden, Germany). Contaminating DNA was removed from RNA samples with TURBO DNA-free (Ambion, United States). The RNA concentration was measured using NanoDrop ND-1000 (Thermo Scientific). The integrity of the RNA samples was analyzed with the BioAnalyzer 2100 (Agilent Technologies) using the RNAnano 6000 kit (Agilent Technologies). Labeling, microarray hybridization, scanning, and data processing were performed in the Genomics Unit of the Centro Nacional de Biotecnología ${ }^{1}$. The data obtained from each LLQR mutant was always compared with the E. coli ATCC 25922 wild-type strain. The FIESTA program was used for analysis $^{2}$ and the $p$-value was determined according to the FDR algorithm ( $p<0.05$ was considered significant). Microarray RNA data are available at GEO $\mathrm{NCBI}^{3}$ (accession number GSE86341).

\footnotetext{
${ }^{1}$ http://wwwuser.cnb.csic.es/ genomica/servicos.htm

${ }^{2}$ http://bioinfogp.cnb.csic.es

${ }^{3}$ http://www.ncbi.nlm.nih.gov/geo/
} 
TABLE 1 | Bacterial strains used in this study and ciprofloxacin susceptibility.

\begin{tabular}{|c|c|c|c|c|}
\hline Strain & Genetic description & CIPa $^{a}$ & Use in this study & Reference \\
\hline E. coli ATCC 25922 & Wild-type E. coli & 0.004 & Transcriptomic assays & 7 \\
\hline EC14 & E. coli ATCC 25922 coding for QnrS1 & 0.125 & Transcriptomic assays & 7 \\
\hline EC19 & E. coli ATCC 25922 with deleted marR gene and coding for QnrS1 & 0.5 & Transcriptomic assays & 7 \\
\hline EC24 & $\begin{array}{l}\text { E. coli ATCC } 25922 \text { with the substitution Ser83Leu in GyrA and coding } \\
\text { for QnrS1 }\end{array}$ & 1 & Transcriptomic assays & 7 \\
\hline ECO4 & $\begin{array}{l}\text { E. coli ATCC } 25922 \text { with the Ser83Leu substitution in GyrA and } \\
\text { Ser80Arg in ParC }\end{array}$ & 0.5 & Viability assays (Killing curves) & 7 \\
\hline EC59 & $\begin{array}{l}\text { E. coli ATCC } 25922 \text { with substitutions Ser83Leu+Asp87Asn in GyrA, } \\
\text { Ser80Arg in ParC, the deleted marR gene and coding for QnrS1 }\end{array}$ & 32 & Viability assays (Killing curves) & 7 \\
\hline E. coli ATCC $25922 \Delta s d h C$ & E. coli ATCC 25922 with sdhC deleted & 0.004 & Viability assays (Killing curves) & This study \\
\hline E. coli ATCC $25922 \Delta$ cyoA & E. coli ATCC 25922 with cyoA deleted & 0.004 & Viability assays (Killing curves) & This study \\
\hline $\mathrm{ECO} 4 \Delta s d h C$ & $\begin{array}{l}\text { E. coli ATCC } 25922 \text { with substitutions Ser83Leu in GyrA and Ser80Arg } \\
\text { in ParC with deleted sdhC }\end{array}$ & 0.5 & Viability assays (Killing curves) & This study \\
\hline EC04 $\triangle c y O A$ & $\begin{array}{l}\text { E. coli ATCC } 25922 \text { with substitutions Ser83Leu in GyrA and Ser80Arg } \\
\text { in ParC with deleted cyoA }\end{array}$ & 0.5 & Viability assays (Killing curves) & This study \\
\hline EC59 $\Delta s d h C$ & $\begin{array}{l}\text { E. coli ATCC } 25922 \text { with substitutions Ser83Leu+Asp87Asn in GyrA, } \\
\text { Ser80Arg in ParC, deleted marR gene and coding for QnrS1 with } \\
\text { deleted sdhC }\end{array}$ & 32 & Viability assays (Killing curves) & This study \\
\hline \multirow[t]{2}{*}{ EC59 $\triangle$ cyoA } & $\begin{array}{l}\text { E. coli ATCC } 25922 \text { with the substitutions Ser83Leu+Asp87Asn in } \\
\text { GyrA, Ser80Arg in ParC, deleted marR gene and coding for QnrS1 }\end{array}$ & 32 & Viability assays (Killing curves) & This study \\
\hline & With deleted cyoA & & & \\
\hline
\end{tabular}

a MIC $(\mu \mathrm{g} / \mathrm{mL})$ of ciprofloxacin.

\section{Annotation of Gene Functions and Regulation}

Gene functions (COG, Clusters of Orthologous Groups) (Tatusov et al., 2000) were identified using the following bioinformatics resources for E. coli: ECOCYC ${ }^{4}$, ECOGENE ${ }^{5}$, Gene Expression Database ${ }^{6}, \mathrm{KEGG}^{7}, \mathrm{BPROM}^{8}$, and DAVID ${ }^{9}$. Venn diagrams were created using the http://bioinfogp.cnb.csic.es/tools/venny/index. html website.

\section{Microarray RNA Data Validation}

Real time RT-PCR was used, as described (Rodríguez-Martínez et al., 2012), to confirm specific transcriptome data ( $s d h C, s u c D$, nouH, recA, focA, tnaA genes), both in presence and absence of ciprofloxacin. The LightCycler FastStart DNA Master SYBR Green I Kit (Roche) was used for amplification. To normalize expression levels, target gene transcripts were calculated relative to the $m d h$ gene, using the $2^{-\Delta \Delta C T}$ method. The primers used for gene amplification are indicated in Supplementary Table 1.

\section{Antimicrobial Susceptibility Testing}

Antimicrobial susceptibility was determined by microdilution according to CLSI guidelines (CLSI). The quinolones tested were ciprofloxacin, norfloxacin, ofloxacin, levofloxacin, moxifloxacin and nalidixic acid (Sigma-Aldrich, Madrid, Spain).

\footnotetext{
${ }^{4} \mathrm{http} / / /$ ecocyc.org/gene?orgid=ECOLI\&id=PD00205\#tab=REGULON

${ }^{5}$ http://ecogene.org/

${ }^{6} \mathrm{https}: / /$ genexpdb.ou.edu/main/

${ }^{7}$ http://www.genome.jp/kegg-bin/get_htext?htext=ko00002\&filedir=\%2fkegg\%

2 fbrite\%2fko\&length=

${ }^{8}$ http://linux1.softberry.com/berry.phtml

${ }^{9}$ https://david.ncifcrf.gov/gene2gene.jsp
}

\section{Time-Kill Curve Assays}

Potentiating antibacterial activity by enhancing microbial ROS production has been demonstrated previously (Brynildsen et al., 2013). To test the impact of ROS modulation (by $s d h C$ or cyoA deletion) on bacterial viability in E. coli ATCC 25922, EC04 and EC59 strains, time-kill curves were assayed in MHB (Mueller-Hinton broth, Becton Dickinson, Le Pont-deClaix, France) at ciprofloxacin and ofloxacin concentrations of 2xMIC (Table 1). Growth in drug-free broth was evaluated in parallel, as a control. Cultures were incubated at $37^{\circ} \mathrm{C}$, and shaken at $180 \mathrm{rpm}$. An initial inoculum of $10^{6} \mathrm{CFU} / \mathrm{mL}$ from a fresh overnight culture was used in all experiments. Bacterial concentrations were determined at $0,1,2,3,4$, and $24 \mathrm{~h}$ by colony counting on drug-free agar.

In parallel, the time courses of E. coli ATCC 25922, EC04 and EC59 cells treated with carboxin $(500 \mu \mathrm{M})$, an inhibitor of succinate dehydrogenase (Brynildsen et al., 2013), and a fluoroquinolone (ciprofloxacin or ofloxacin at $2 x M I C$ ), were compared with treatment with fluoroquinolones alone. Carboxin (Sigma-Aldrich, Madrid, Spain) was dissolved in 100\% ethanol (Brynildsen et al., 2013).

\section{RESULTS}

\section{Global Expression Profiles}

In order to establish the role of LLQR phenotypes on the differential expression in response to quinolones, the expression profiles of isogenic E. coli carrying LLQR harboring frequent chromosomal and PMQR mechanisms (EC14 coding for QnrS1; EC19 with deleted marR gene and coding for QnrS1; and EC24 with the Ser83Leu substitution in GyrA and coding 


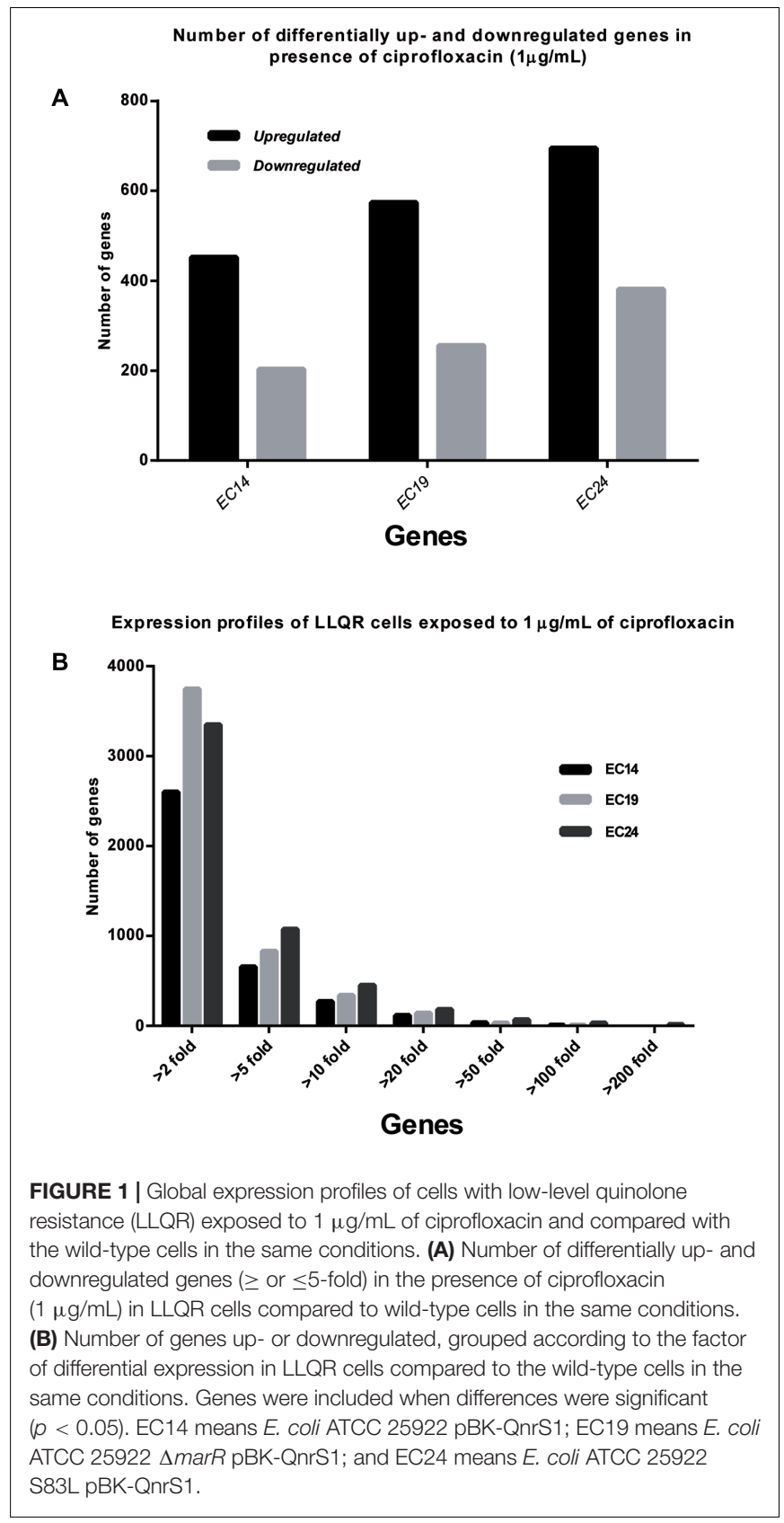

for QnrS1) (Machuca et al., 2014) were evaluated in the presence of significant clinical concentrations of ciprofloxacin (1 $\mu \mathrm{g} / \mathrm{mL}$, the breakpoint for reduced susceptibility according to CLSI and near to $1 / 2$ of serum Cmax) (Mandell et al., 2010; Clinical and Laboratory Standards Institute [CLSI], 2016) and compared with those of wild-type E. coli ATCC 25922 (Figure 1 and Supplementary Tables 2, 3). The MIC values of ciprofloxacin for these susceptible LLQR strains (EC14, EC19, EC24) were 32-, 125- and 250-fold higher compared to the wild-type strain. As expected, significant differences were observed in the numbers of differentially up- and downregulated genes $(>5$-fold, $p<0.05)$. Most genes were upregulated and the differences were proportional to the MIC values for strains with LLQR (EC14: 452/203 genes; EC19: 574/256 genes; EC24: 695/381 genes, upregulated and downregulated, respectively) (Figure 1A). Expression of $>2500$ genes, whether by upregulation or downregulation, varied more than $>2$-fold, reflecting the marked differential response to ciprofloxacin in LLQR strains. Under these this clinically significant concentration of ciprofloxacin, variations of $>10$-fold for EC14/EC19/EC24 (271/339/450 genes), of $>50$-fold (34/32/68 genes) and of $>100$-fold $(8 / 8 / 33$ genes) were observed (Figure 1B). Just a few genes had differential expression of more than 200-fold for LLQR cells compared to the wild-type (16 genes for EC24).

Figure 2 shows gene expression classified according to the COG functional categories (Tatusov et al., 2000) (showing values equal to or more than 30 -fold, that is, large differences, $p$-value $<0.05$ ) (see also Supplementary Table 3). Among these genes, four functional groups were chiefly affected, related to the number of genes involved. "Energy production and conversion genes (EPC)" and "Amino acid metabolism and transport genes (AMT)" tended to be genes upregulated at higher levels ( $\geq 30$-fold). These differences were proportional to MIC values for strains with LLQR (EC14: 9/4 genes; EC19: 15/11 genes; EC24: 41/24 genes for EPC and AMT genes, respectively) (Figure 2). A reduction of genes related to "Bacteriophage Activation (BA)" was observed counter to MIC values for LLQR strains (mostly upregulated in EC14 and EC19) (Figure 2), while there was a considerable number of highly altered genes without functional prediction (both up- and downregulated).

Figure 3 shows differentially expressed genes equal to or greater than 30-fold ( $p$-value $<0.05$ ) (regarded as major differences in terms of expression). Interestingly, the strains with LLQR were proportionally reorganized in response to ciprofloxacin stress (Figure 3). In this regard, 141 genes showed altered expression for at least one strain with LLQR, and $14.9 \%$ of these were shared in all three strains. In total, under ciprofloxacin-induced stress, 25 and 47 genes were specifically affected in EC14 and EC24, respectively, while neither of these strains shared any genes with altered expression. EC19, on the other hand, shared 20 and 27 genes with EC14 and EC24, respectively. These data indicate that, with respect to EC14 and EC24, EC19 is at a transitional stage in its response to ciprofloxacin (see also Supplementary Table 3).

The transcriptome microarray data for six specific genes was validated by quantitative RT-PCR, confirming both the upregulation and downregulation results in presence of ciprofloxacin (Supplementary Table 1). No significant differences were observed for these genes in absence of ciprofloxacin (data not shown). No significant differences were observed when E. coli ATCC 25922 and EC14 were compared in absence of ciprofloxacin (data not shown).

\section{Increased ROS Modulation in Isogenic E. coli with LLQR}

Multiple genes implicated in oxidative metabolism and modulation of ROS [related to the TCA cycle ( $s d h C$ : Succinate dehydrogenase; pta: Phosphotransacetylase; sucA-D: Succinyl 


\section{Functional classification}

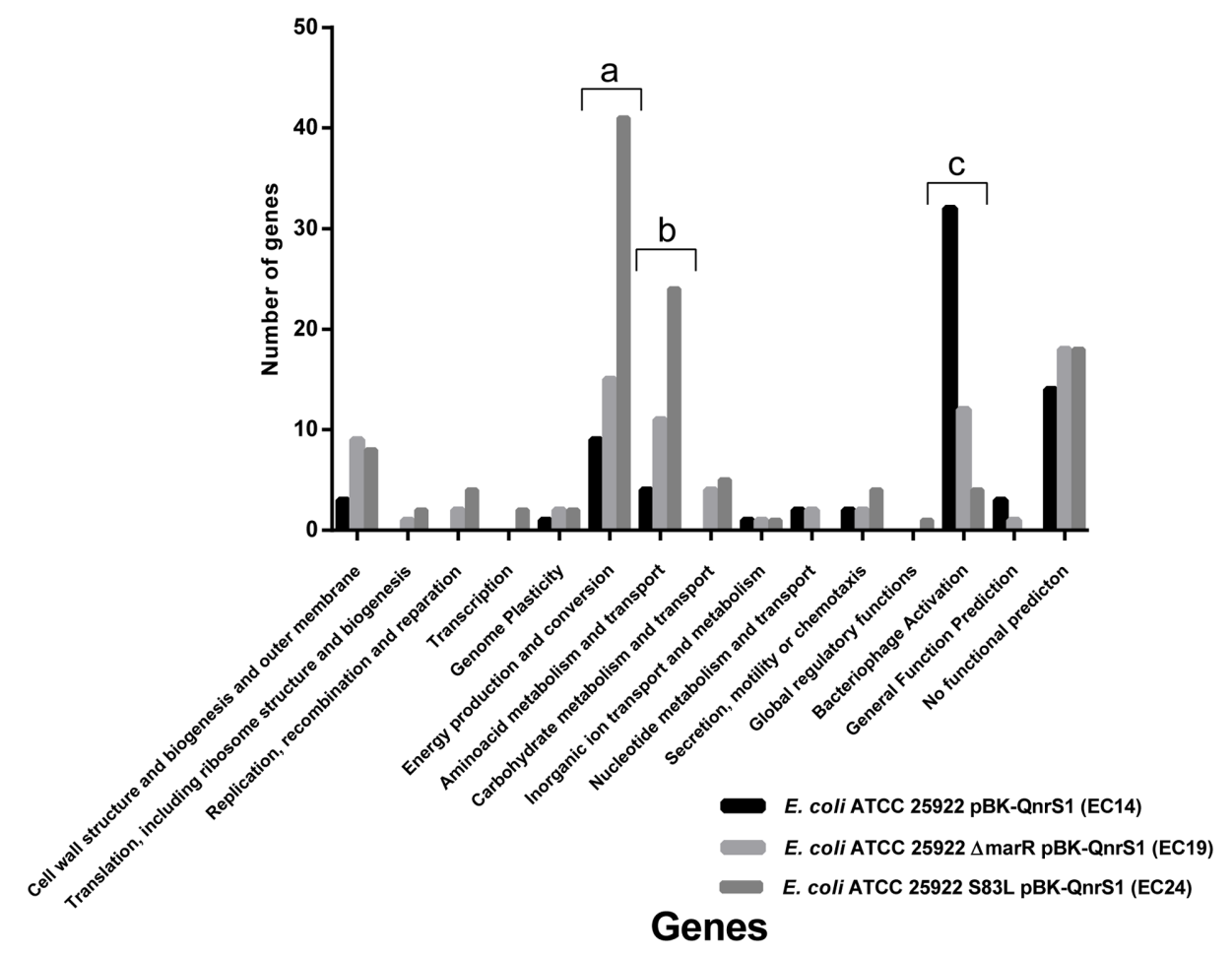

FIGURE 2 | Gene expression classified by COG (Cluster of Orthologous Groups) (Tatusov et al., 2000) for functional categories (greater than or equal to 30 -fold, $p$-value $<0.05)$.

CoA synthase complex; up to 495-fold), aerobic respiration (cyoA-E: Cytochrome oxidase; nuoG or nuoH: Ubiquinone oxidoreductase; atpH: ATP synthase; up to 108-fold), glucose metabolism (gnd: Gluconate-P dehydrogenase; $z w f$ : Glucose-6-phosphate 1-dehydrogenase; up to 5.5-fold) or detoxification systems (dps: Stress-induced Fe-binding, ahpC: Alkyl hydroperoxide reductase, sodB: Superoxide dismutase, kat $G$ : Catalase-peroxidase; up to 12.4-fold)] were highly upregulated in LLQR mutants (Figure 4 and Supplementary Table 2). Proteome profiling based on LC-MS/MS (liquid chromatography-mass spectrometry) showed a similar trend with proteins related to ROS modulation over-represented in LLQR mutants (Supplementary Table 4). Differences observed were proportional to MIC values for strains with LLQR. These data indicate that strains with LLQR would produce lower levels of ROS in the presence of clinical concentrations of ciprofloxacin (Brynildsen et al., 2013; Dwyer et al., 2014).

\section{Moderate SOS Response in Isogenic E. coli with LLQR}

Genes involved in DNA repair and the SOS response are highly upregulated in response to quinolones (Dwyer et al., 2007; Baharoglu and Mazel, 2014). In our experiment, the relative concentrations of ciprofloxacin used were 250xMIC for E. coli ATCC 25922, 8xMIC for EC14, 2xMIC for EC19 and 1xMIC for EC24. Under this clinically significant concentration of ciprofloxacin, a clear reduction of SOS system gene expression was observed in strains with LLQR [related to SOS system activation ( $r e c A$ : the multifunctional DNA recombination and repair protein and master regulator of the SOS system; up to -30.7-fold), DNA repair by homologous recombination (ruvA: Holliday junction recognition protein; up to -4.75 -fold), DNA nucleotide excision repair ( $u v r B$ : Excision nuclease subunit B; up to -20.3 -fold) or DNA repair by translesion synthesis (umuC: Translesion DNA polymerase V; up to -7.9-fold)] (Figure 5 and Supplementary Table 2).

\section{Impact of LLQR Phenotypes on Other Bacterial Survival Systems}

The PCD and TA systems have been linked to cell death in response to quinolones (Erental et al., 2012, 2014). We analyzed patterns of expression of multiple TA systems (both type I and type II) and found both upregulated ( $g n s A, y m c E, y j h Q, y j h X$, or yihE) and downregulated genes (hokA, hokD, symE, chpBK, yhaV, yef $M$, yoeB, or $y f j F$ ) in strains with LLQR (Yamaguchi and Inouye, 2011). Interestingly, the $y i h E$ gene (coding for a key kinase regulator that protects $E$. coli from antimicrobials like quinolones by antagonizing the MazEF TA module) was upregulated in LLQR strains (Supplementary Figure 1 and Table 2) (DorseyOresto et al., 2013).

Altered expression of downstream genes linked to the mazEF pathway involved in PCD control was also detected 


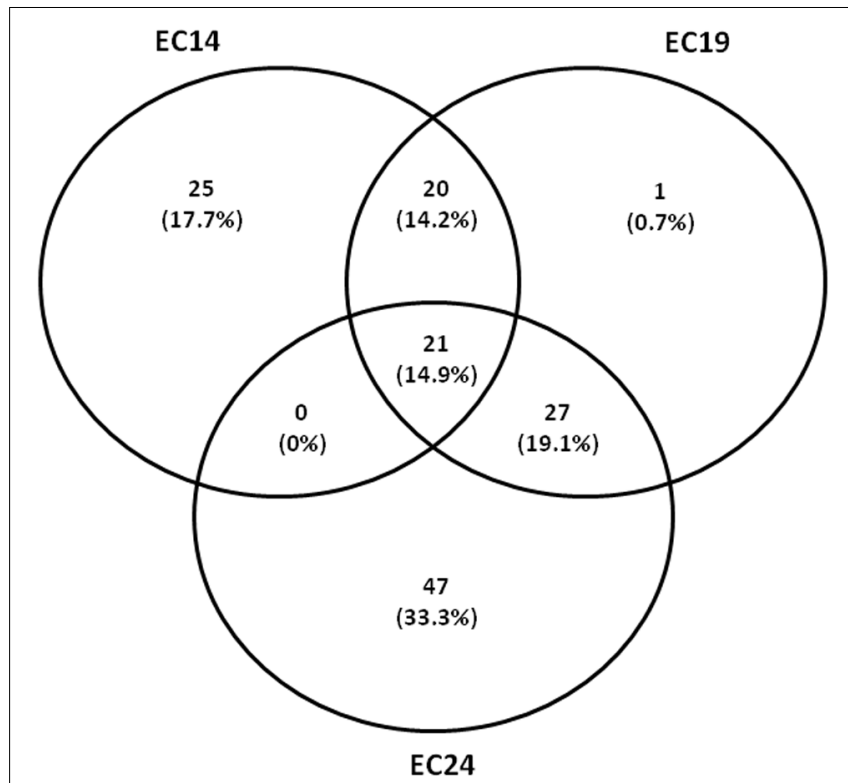

FIGURE 3 | Overlap between differently expressed genes (greater than or equal to 30 -fold, $p$-value $<0.05$ ) on exposure to ciprofloxacin $(1 \mu \mathrm{g} / \mathrm{mL})$ between low-level quinolone resistant (LLQR) cells compared to the wild-type cells in the same conditions. Venn diagram shows the overlap. The numbers indicated on the diagram refer to the number of genes with significantly altered expression levels in one or both conditions. LLQR phenotypes: EC14 means E. coli ATCC 25922 pBK-QnrS1; EC19 means E. coli ATCC 25922 $\Delta$ marR pBK-QnrS1; and EC24 means E. coli ATCC 25922 S83L pBK-QnrS1.

(Erental et al., 2014). $y f i D, c l p X, c l p P$, and $y f b U$ were upregulated; $s l y D$ and $y g c R$ were downregulated in strains with LLQR. Interestingly, genes for the major quinolone target in Gram-negative bacteria, the DNA gyrase subunits, tended to be downregulated (gyrA: DNA gyrase, subunit A, up to -7.18 -fold and gyrB: DNA gyrase, subunit B, up to -8.9 -fold) in strains with LLQR. Finally, several genes implicated in the DNA mismatch repair system tended to be downregulated ( $m u t H$ : up to -2.84 -fold; $m u t L$ : up to -2.14 -fold; $m u t M$ : up to -5.57-fold; mutY: up to -4.03 -fold) in LLQR strains. mutS was not significantly affected (Supplementary Figure 1 and Table 2).

\section{Sensitization of LLQR Phenotypes by Targeting Oxidative Metabolism}

The transcriptomic data obtained indicated that oxidative metabolism (related to the endogenous production of ROS and detoxification systems) plays an important and differential role in quinolone response in strains with LLQR. We tested the impact of $s d h C$ (Succinate dehydrogenase component) and cyoA (Cytochrome $\mathrm{O}$ oxidase component) deletions on bacterial viability. Inhibiting these two targets has previously been shown to enhance endogenous microbial ROS production and potentiate the antibacterial activity of quinolones in full susceptible wild-type E. coli (Brynildsen et al., 2013). Here, we tested its putative role in terms of LLQR phenotype sensitization. In terms of time-kill curves (assayed in MHB at ciprofloxacin and ofloxacin concentrations of $2 x M I C), s d h C$ deletion increased sensitivity to fluoroquinolones (both ciprofloxacin and ofloxacin) in wild-type and LLQR strains (Figure 6). A marked reduction in viable bacteria counts was observed for ciprofloxacin after a short incubation (E. coli ATCC 25922: $\Delta \log =1.4$, EC04: $\Delta \log =1.5$, EC59: $\Delta \log =0.4$; after $4 \mathrm{~h}$ ) and a long one (E. coli ATCC 25922: $\Delta \log =0.6, \mathrm{EC} 04: \Delta \log =1.4, \mathrm{EC} 59: \Delta \log =2.3$; after $24 \mathrm{~h}$ ). Although it has been suggested that $c y o A$ is implicated in the quinolone sensitization process in E. coli (Brynildsen et al., 2013), only a minor impact was observed in our study in terms of expression (data not shown).

We also wondered whether chemical inhibition of this validated target $(s d h C)$ would increase sensitivity to these bactericidal antimicrobial agents. We treated wild-type and LLQR strains with carboxin, an inhibitor of succinate dehydrogenase (Brynildsen et al., 2013), and measured their susceptibility to ciprofloxacin and ofloxacin, respectively. The addition of carboxin alone had no effect on the growth of wild-type cells (data not shown). However, wild-type and LLQR cells treated with fluoroquinolones and carboxin demonstrated increased sensitivity compared to cells treated with fluoroquinolones alone. For ciprofloxacin, this reduction in viability was observed after a short period of incubation (E. coli ATCC 25922: $\Delta \log =1.1, \mathrm{EC} 04: \Delta \log =0.9, \mathrm{EC} 59: \Delta \log =0.6$; after $4 \mathrm{~h}$ ) and a long one (E. coli ATCC 25922: $\Delta \log =0.6, \mathrm{EC} 04$ : $\Delta \log =0.3$, EC59: $\Delta \log =2.2$; after 24 h) (Figure 6).

\section{DISCUSSION}

Using a transcriptomic approach, we provided evidence of the differential response at clinically significant concentrations of ciprofloxacin $(1 \mu \mathrm{g} / \mathrm{mL}$, the breakpoint for reduced susceptibility according to CLSI and near to $1 / 2$ of serum Cmax) (Mandell et al., 2010) in E. coli strains with LLQR harboring frequent quinolone resistance mechanisms (chromosomal and plasmid-mediated). This reduced susceptibility observed in LLQR phenotypes (directly related to MIC value and independently of the subjacent molecular mechanism) led to modulation of multiple systems contributing to bacterial survival under this therapeutic concentration. Additionally, this approach resulted to be useful to start the study of new potential targets for LLQR strains sensitization by targeting ROS modulation.

Ciprofloxacin caused a major reprogramming of gene expression across the genome: hundreds of genes exhibited upregulation or downregulation (Figures 1, 2 and Supplementary Tables 2, 3), supporting previous literature for quinolones and other antimicrobials (Shaw et al., 2003; Peter et al., 2004; Kohanski et al., 2007, 2010; Ferrándiz et al., 2016). Although it has been reported that both specific GyrA substitutions (Asp87Gly), marR gene deletion or $q n r S$ expression may modify moderately the expression of several genes in bacteria (Seoane and Levy, 1995; Okumura et al., 2011; Kwak et al., 2013; Webber et al., 2013), this aspect had not a relevant impact in the interpretation of our results. Phenotypes with LLQR possessed a marked differential response to ciprofloxacin, and the differences observed were proportional to the MIC values for LLQR strains. One of the processes most affected was related to oxidative 


\section{Upregulated oxidative metabolism in LLQR isogenic E. coli}

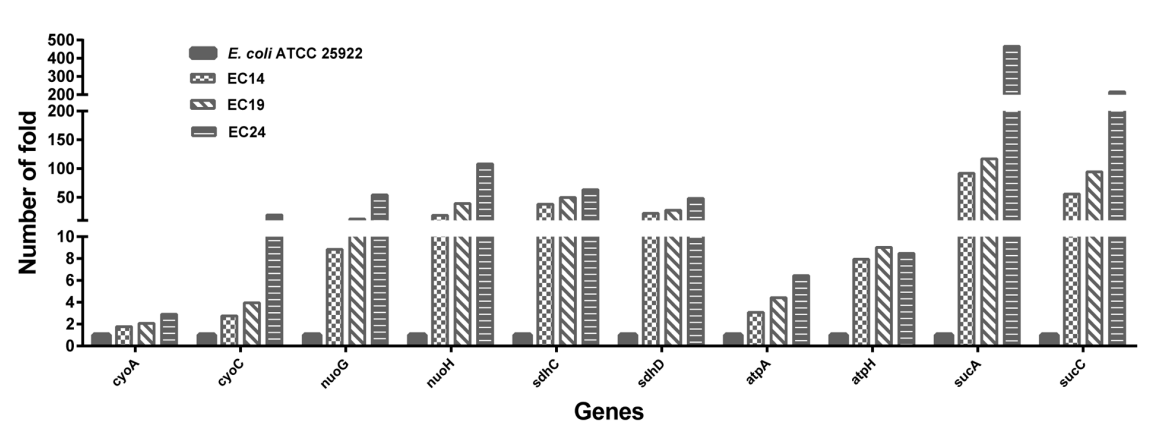

Upregulated detoxification systems in LLQR isogenic $E$. coli

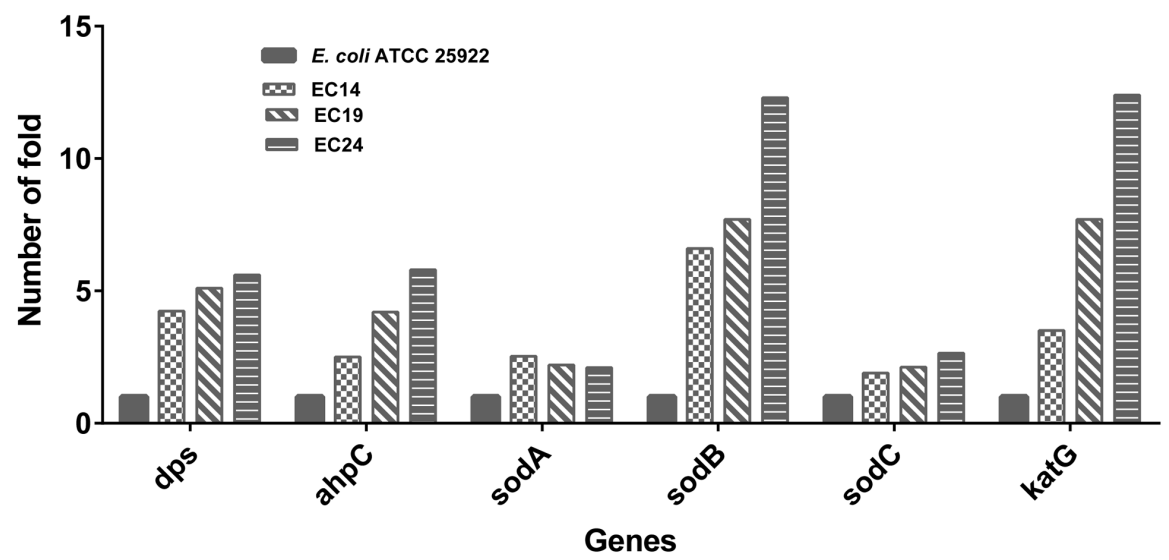

FIGURE 4 | Impact on ROS response of low-level quinolone-resistant (LLQR) cells exposed to $1 \mu \mathrm{g} / \mathrm{mL}$ of ciprofloxacin compared to wild-type cells in the same conditions. LLQR phenotypes: EC14 means E. coli ATCC 25922 pBK-QnrS1; EC19 means E. coli ATCC 25922 AmarR pBK-QnrS1; and EC24 means E. coli ATCC 25922 S83L pBK-QnrS1. All indicated genes show a significantly different pattern of expression between LLQR strains and wild-type $E$. coli ( $p$-value $<0.05)$. Standard deviations were within $10 \%$ of the means.

\section{Decreased SOS system expression in LLQR isogenic E. coli}

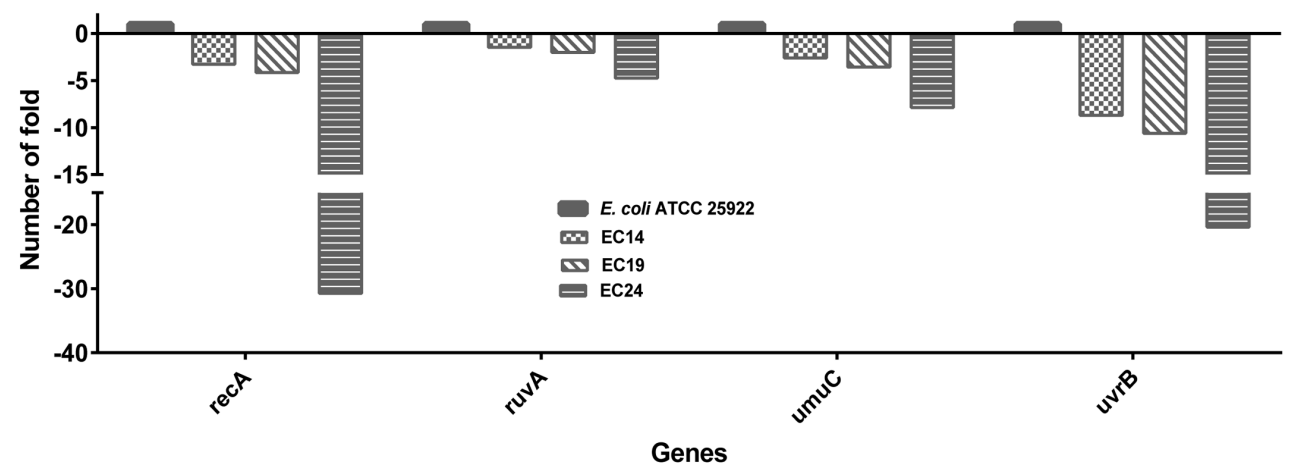

FIGURE 5 | Impact on SOS response of low-level quinolone-resistant (LLQR) cells exposed to $1 \mu \mathrm{g} / \mathrm{mL}$ of ciprofloxacin, compared to wild-type cells in the same conditions. LLQR phenotypes: EC14 means E. coli ATCC 25922 pBK-QnrS1; EC19 means E. coli ATCC 25922 AmarR pBK-QnrS1; and EC24 means E. coli ATCC 25922 S83L pBK-QnrS1. All indicated genes shown a pattern of expression significantly different among LLQR strains and wild-type $E$. coli (p-value $<0.05)$. Standard deviations were within $10 \%$ of the means. 


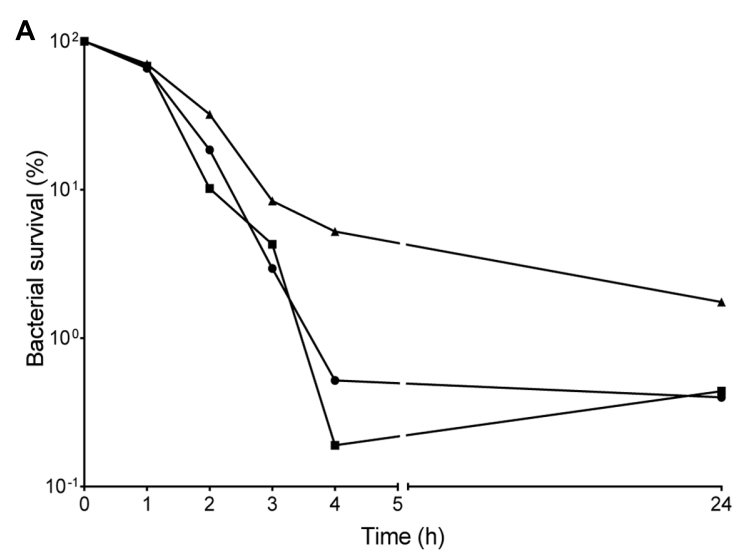

$\star$ ATCC $25922 *$ ATCC $25922 \Delta$ sdhC * ATCC $25922+$ Carboxin

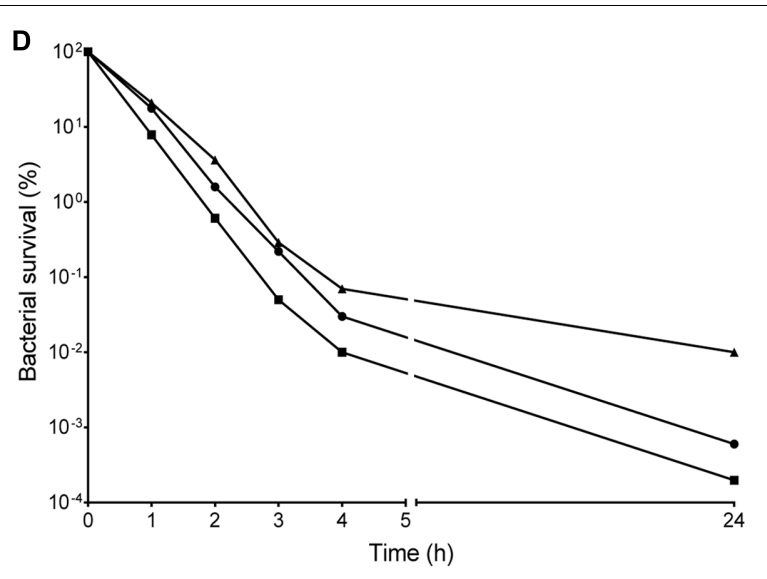

- ATCC 25922 - ATCC 25922 ssdhC * ATCC $25922+$ Carboxin
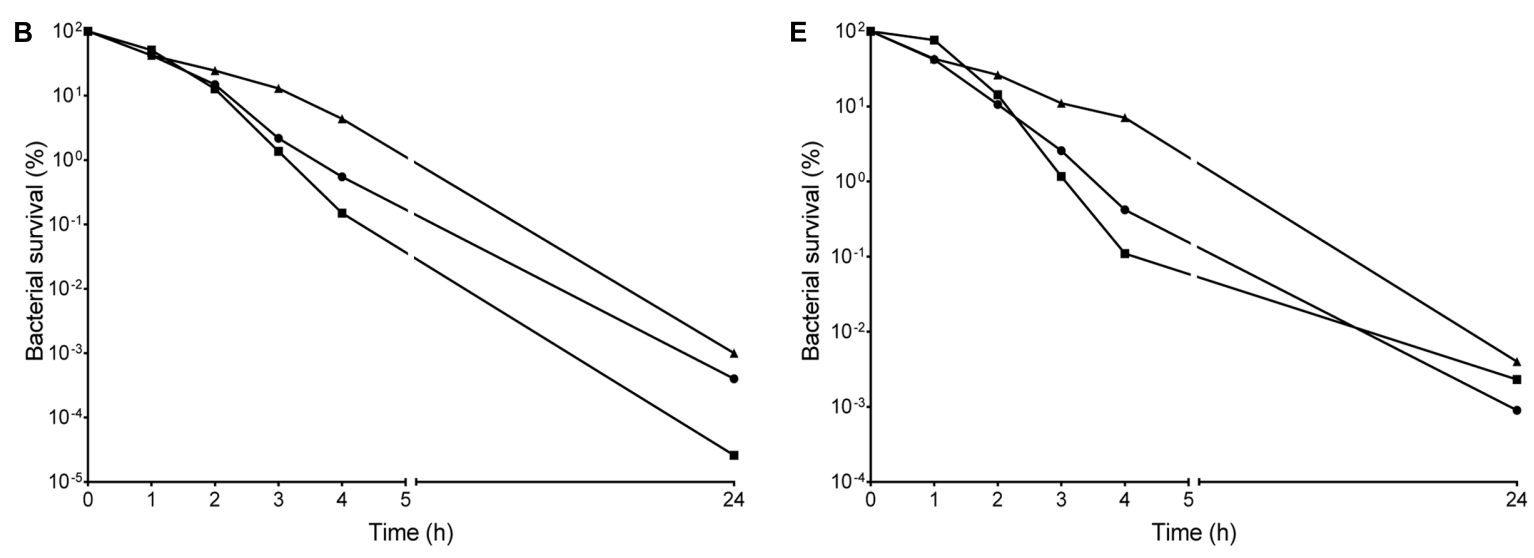

$-\mathrm{ECO} 4$ EC04 $\Delta \mathrm{sdhC} \bullet \mathrm{ECO} 4+$ Carboxin

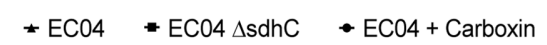

C

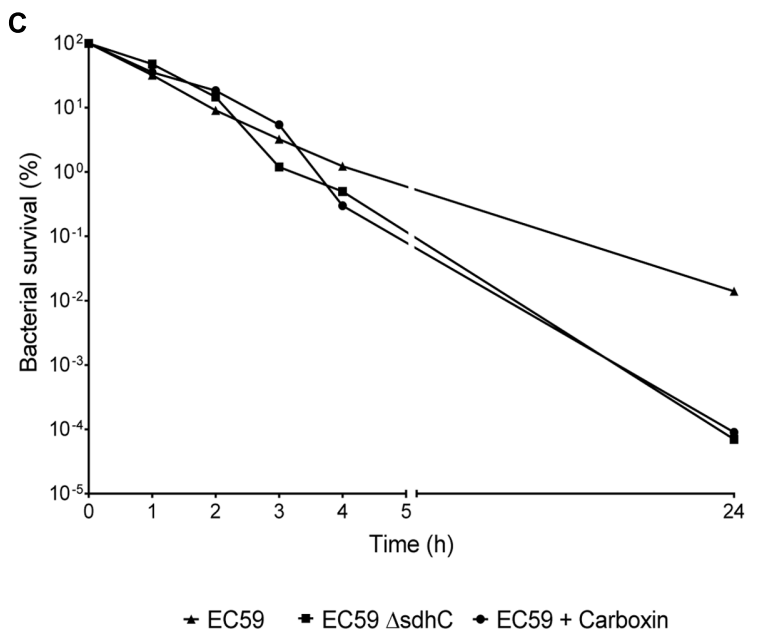

$\mathbf{F}$

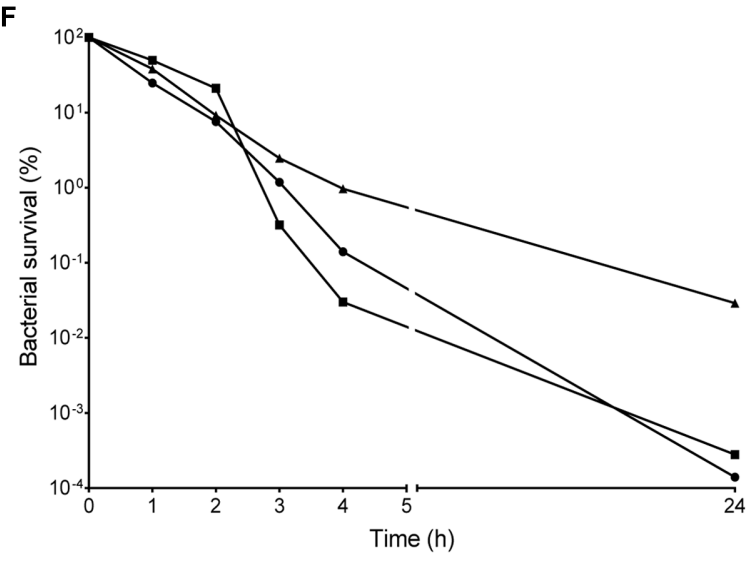

- EC59 *E59 $\Delta$ sdhC $*$ EC59 + Carboxin

FIGURE 6 | Evaluation of susceptibility to killing by ciprofloxacin (A-C), ofloxacin (D-F), and combination treatments with carboxin (500 $\mu$ M). Fluoroquinolone concentrations tested were equivalent to $2 x M I C$ values. Data are plotted as mean \pm standard deviation. 
metabolism. It has already been shown that the antibacterial activity of antibiotics such as quinolones can be increased by potentiating endogenous microbial ROS production (Wang and Zhao, 2009; Brynildsen et al., 2013). Our results are consistent with these studies and indicate that strains with LLQR would produce lower levels of ROS (Brynildsen et al., 2013; Dwyer et al., 2014). At clinically significant concentrations of ciprofloxacin, the quinolone-induced complex redox alterations downstream of their target-specific interactions that contribute to cellular damage and death would be reduced in strains with LLQR (Erental et al., 2012; Brynildsen et al., 2013; Dorsey-Oresto et al., 2013; Dwyer et al., 2014; Händel et al., 2014, 2016; Méhi et al., 2014; Ferrándiz et al., 2016). In this context, resistance would be the result on interaction at the genetic and gene expression levels (Händel et al., 2014; Freihofer et al., 2016).

In E. coli, RecA-LexA coordinates the DNA damage response that allows two opposing responses: life, mediated by the SOS; and death, mediated by the PCD (Baharoglu and Mazel, 2014; Erental et al., 2014). The choice seems to depend on the degree of DNA damage to the cell (and is indirectly a function of MIC values) (Rodríguez-Martínez et al., 2016a). At $1 \mu \mathrm{g} / \mathrm{mL}$ of ciprofloxacin, LLQR strains would be affected by stress caused by moderate DNA damage compared to the stress in wild-type E. coli caused by a severe DNA damage. Moderate SOS response in LLQR strains could lead to survival, while massive DNA damage in wild-type E. coli could lead to dead population under exacerbated SOS response (Erental et al., 2014). At clinically significant concentrations of ciprofloxacin, the evolution to clinical resistance (ciprofloxacin MIC higher than $2 \mu \mathrm{g} / \mathrm{mL}$ ) would be favored in the strain with LLQR, allowing survival and favoring processes of recombination and DNA repair, such as translesion synthesis (Morgan-Linnell and Zechiedrich, 2007; Morgan-Linnell et al., 2009; Baharoglu and Mazel, 2014; Erental et al., 2014). These data would support the higher frequency of mutants observed in LLQR strains (Briales et al., 2011; Machuca et al., 2014).

Furthermore, the expression profile was clearly altered in these LLQR strains, and involved even TA system genes, PCD genes and MMR genes. Interestingly, the yihE gene (coding for a key kinase regulator that protects E. coli from antimicrobials like quinolones by antagonizing the MazEF TA module) was upregulated in LLQR strains (Dorsey-Oresto et al., 2013), increasing the level of protection against PCD processes. Downregulation of the DNA gyrase genes (both gyrA and gyrB) also inferred the differential degree of stress in strains with LLQR compared to the wild-type strains (Supplementary Figure 1).

The redox stress component of antibiotic lethality contributes to cell death (usually without MICs values modification) (Wang and Zhao, 2009; Brynildsen et al., 2013). More specifically, antimicrobials like quinolones produce alterations to the central metabolism, cellular respiration and iron metabolism initiated by drug-mediated disruptions of target-specific processes and resulting in cellular damage (Dwyer et al., 2014; Zhao and Drlica, 2014; Lobritz et al., 2015; Zhao et al., 2015). Drug tolerance in pathogenic clinical isolates involves mutations in the oxidative stress response and detoxifying genes (McMurry et al., 1998; Koutsolioutsou et al., 2005; Páez et al., 2010; Thomas et al., 2013). Our transcriptomic data indicated that oxidative metabolism (related both to endogenous ROS modulation and detoxification systems) plays an important differential role in the response of quinolones in LLQR strains. Here we hypothesized that, against quinolones, the deletion or inhibition of the central components of the TCA cycle would lead to sensitization in strains with LLQR similar to that observed in fully susceptible wild-type E. coli, as previously described (Brynildsen et al., 2013).

Our results show that inhibiting succinate dehydrogenase (predictable according to transcriptomic comparisons) is sufficient to increase sensitivity to quinolones in LLQR E. coli. Although carboxin may not be useful as an antibiotic adjuvant because of its toxicity ${ }^{10}$, the data reinforce the potential usefulness of this strategy.

In summary, the global response to ciprofloxacin is significantly altered in LLQR E. coli and affects critical systems for survival and the emergence of antimicrobial resistance at clinically relevant concentrations. This approach validated ROS modulation as an interesting target in bacterial re-sensitization after drug resistance development.

\section{AUTHOR CONTRIBUTIONS}

J-MR-M and AP designed the study. J-MR-M, JM, ER, AB, and PD-d-A performed transcriptomic assays, further analysis and viability assays. J-MR-M, AP, and JB. contributed ideas and edited the manuscript. All authors read, commented on, and approved the final manuscript.

\section{ACKNOWLEDGMENTS}

This work was supported by the Ministerio de Sanidad y Consumo, Instituto de Salud Carlos III (projects PI11-00934 and PI14/00940) and the Consejería de Innovación, Ciencia y Empresa, Junta de Andalucía (P11-CTS-7730), Spain, by the Plan Nacional de I+D+i 2008-2011 and the Instituto de Salud Carlos III, Subdirección General de Redes y Centros de Investigación Cooperativa, Ministerio de Economía y Competitividad, the Spanish Network for Research in Infectious Diseases (REIPI RD12/0015)—co-financed by European Development Regional Fund 'A way to achieve Europe' ERDF.

\section{SUPPLEMENTARY MATERIAL}

The Supplementary Material for this article can be found online at: http://journal.frontiersin.org/article/10.3389/fmicb. 2017.01370/full\#supplementary-material

\footnotetext{
${ }^{10} \mathrm{https} / / /$ www.epa.gov/sites/production/files/2015-07/documents/epa-507.pdf
} 


\section{REFERENCES}

Baharoglu, Z., and Mazel, D. (2014). SOS, the formidable strategy of bacteria against aggressions. FEMS Microbiol. Rev. 38, 1126-1145. doi: 10.1111/15746976.12077

Baym, M., Stone, L. K., and Kishony, R. (2016). Multidrug evolutionary strategies to reverse antibiotic resistance. Science 351:aad3292. doi: 10.1126/science.aad3292

Blair, J. M. A., Webber, M. A., Baylay, A. J., Ogbolu, D. O., and Piddock, L. J. V. (2015). Molecular mechanisms of antibiotic resistance. Nat. Rev. Microbiol. 13, 42-51. doi: 10.1038/nrmicro3380

Briales, A., Rodriguez-Martinez, J. M., Velasco, C., Diaz de Alba, P., DominguezHerrera, J., Pachon, J., et al. (2011). In vitro effect of $q n r A 1$, qnrB1, and qnrS1 genes on fluoroquinolone activity against isogenic Escherichia coli isolates with mutations in gyrA and parC. Antimicrob. Agents Chemother. 55, 1266-1269. doi: 10.1128/AAC.00927-10

Brynildsen, M. P., Winkler, J. A., Spina, C. S., MacDonald, I. C., and Collins, J. J. (2013). Potentiating antibacterial activity by predictably enhancing endogenous microbial ROS production. Nat. Biotechnol. 31, 160-165. doi: 10.1038/nbt.2458

Clinical and Laboratory Standards Institute [CLSI] (2016). Performance Standards for Antimicrobial Susceptibility Testing: Twenty-sixth Informational Supplement M100-S26. Wayne, PA: CLSI.

Datsenko, K. A., and Wanner, B. L. (2000). One-step inactivation of chromosomal genes in Escherichia coli K-12 using PCR products. Proc. Natl. Acad. Sci. U.S.A. 97, 6640-6645. doi: 10.1073/pnas.120163297

Dorsey-Oresto, A., Lu, T., Mosel, M., Wang, X., Salz, T., Drlica, K., et al. (2013). YihE kinase is a central regulator of programmed cell death in bacteria. Cell Rep. 3, 528-537. doi: 10.1016/j.celrep.2013.01.026

Drlica, K., Hiasa, H., Kerns, R., Malik, M., Mustaev, A., and Zhao, X. (2009). Quinolones: action and resistance updated. Curr. Top. Med. Chem. 9, 981-998. doi: 10.2174/156802609789630947

Dwyer, D. J., Belenky, P. A., Yang, J. H., MacDonald, I. C., Martell, J. D., Takahashi, N., et al. (2014). Antibiotics induce redox-related physiological alterations as part of their lethality. Proc. Natl. Acad. Sci. U.S.A. 111, E2100-E2109. doi: 10.1073/pnas.1401876111

Dwyer, D. J., Kohanski, M. A., Hayete, B., and Collins, J. J. (2007). Gyrase inhibitors induce an oxidative damage cellular death pathway in Escherichia coli. Mol. Syst. Biol. 3, 91. doi: 10.1038/msb4100135

Erental, A., Kalderon, Z., Saada, A., Smith, Y., and Engelberg-Kulka, H. (2014). Apoptosis-Like Death, an Extreme SOS Response in Escherichia coli. MBio 5, e01426-14. doi: 10.1128/mBio.01426- 14

Erental, A., Sharon, I., and Engelberg-Kulka, H. (2012). Two programmed cell death systems in Escherichia coli: an apoptotic-like death is inhibited by the mazEF-mediated death pathway. PLoS Biol. 10:e1001281. doi: 10.1371/journal. pbio. 1001281

Ferrándiz, M. J., Martín-Galiano, A. J., Arnanz, C., Zimmerman, T., and de la Campa, A. G. (2016). Reactive oxygen species contribute to the bactericidal effects of the fluoroquinolone moxifloxacin in Streptococcus pneumoniae. Antimicrob. Agents Chemother. 60, 409-417. doi: 10.1128/AAC.02299-15

Freihofer, P., Akbergenov, R., Teo, Y., Juskeviciene, R., Andersson, D. I., and Böttger, E. C. (2016). Nonmutational compensation of the fitness cost of antibiotic resistance in mycobacteria by overexpression of tlyA rRNA methylase. RNA 22, 1836-1843. doi: 10.1261/rna.057257.116

Händel, N., Hoeksema, M., Freijo Mata, M., Brul, S., and ter Kuile, B. H. (2016). Effects of stress, reactive oxygen species, and the SOS response on de novo acquisition of antibiotic resistance in Escherichia coli. Antimicrob. Agents Chemother. 60, 1319-1327. doi: 10.1128/AAC.02684-15

Händel, N., Schuurmans, J. M., Feng, Y., Brul, S., and ter Kuile, B. H. (2014). Interaction between mutations and regulation of gene expression during development of de novo antibiotic resistance. Antimicrob. Agents Chemother. 58, 4371-4379. doi: 10.1128/AAC.02892-14

Hooper, D. C., and Jacoby, G. A. (2015). Mechanisms of drug resistance: quinolone resistance. Ann. N. Y. Acad. Sci. 1354, 12-31. doi: 10.1111/nyas.12830

Jacoby, G. A., Strahilevitz, J., and Hooper, D. C. (2014). Plasmidmediated quinolone resistance. Microbiol. Spectr. 22, 664-689. doi: 10.1128/microbiolspec.PLAS-0006-2013

Kohanski, M. A., DePristo, M. A., and Collins, J. J. (2010). Sublethal antibiotic treatment leads to multidrug resistance via radical-induced mutagenesis. Mol. Cell 37, 311-320. doi: 10.1016/j.molcel.2010.01.003
Kohanski, M. A., Dwyer, D. J., Hayete, B., Lawrence, C. A., and Collins, J. J. (2007). A common mechanism of cellular death induced by bactericidal antibiotics. Cell 130, 797-810. doi: 10.1016/j.cell.2007.06.049

Koutsolioutsou, A., Peña-Llopis, S., and Demple, B. (2005). Constitutive soxR mutations contribute to multiple-antibiotic resistance in clinical Escherichia coli isolates. Antimicrob. Agents Chemother. 49, 2746-2752. doi: 10.1128/AAC.49.7. 2746-2752.2005

Kwak, Y. G., Jacoby, G. A., and Hooper, D. C. (2013). Induction of plasmid-carried qnrS1 in Escherichia coli by naturally occurring quinolones and quorum-sensing signal molecules. Antimicrob. Agents Chemother. 57, 4031-4034. doi: 10.1128/ AAC.00337-13

Lobritz, M. A., Belenky, P., Porter, C. B. M., Gutierrez, A., Yang, J. H., Schwarz, E. G., et al. (2015). Antibiotic efficacy is linked to bacterial cellular respiration. Proc. Natl. Acad. Sci. U.S.A. 112, 8173-8180. doi: 10.1073/pnas.1509743112

Machuca, J., Briales, A., Labrador, G., Díaz-de-Alba, P., López-Rojas, R., DocoboPérez, F., et al. (2014). Interplay between plasmid-mediated and chromosomalmediated fluoroquinolone resistance and bacterial fitness in Escherichia coli. J. Antimicrob. Chemother. 69, 3203-3215. doi: 10.1093/jac/dku308

Mandell, G. L., Bennet, J. E., and Dolin, R. (2010). Mandell, Douglas, and Bennett's Principles, and Practice of Infectious Diseases. Philadelphia, PA: Churchill Livingstone Press, 492.

McMurry, L. M., Oethinger, M., and Levy, S. B. (1998). Overexpression of marA, soxS, or $a c r A B$ produces resistance to triclosan in laboratory and clinical strains of Escherichia coli. FEMS Microbiol. Lett. 166, 305-309.

Méhi, O., Bogos, B., Csörgõ, B., Pál, F., Nyerges, A., Papp, B., et al. (2014). Perturbation of iron homeostasis promotes the evolution of antibiotic resistance. Mol. Biol. Evol. 31, 2793-2804. doi: 10.1093/molbev/msu223

Morgan-Linnell, S. K., Boyd, L. B., Steffen, D., and Zechiedrich, L. (2009). Mechanisms accounting for fluoroquinolone resistance in Escherichia coli clinical isolates. Antimicrob. Agents Chemother. 53, 235-241. doi: 10.1128/AAC. 00665-08

Morgan-Linnell, S. K., and Zechiedrich, L. (2007). Contributions of the combined effects of topoisomerase mutations toward fluoroquinolone resistance in Escherichia coli. Antimicrob. Agents Chemother. 51, 4205-4208. doi: 10.1128/ AAC.00647-07

Okumura, R., Liao, C. H., Gavin, M., Jacoby, G. A., and Hooper, D. C. (2011). Quinolone induction of qnrVS1 in Vibrio splendidus and plasmid-carried qnrS1 in Escherichia coli, a mechanism independent of the SOS system. Antimicrob. Agents Chemother. 55, 5942-5945. doi: 10.1128/AAC.05142-11

Páez, P. L., Becerra, M. C., and Albesa, I. (2010). Antioxidative mechanisms protect resistant strains of Staphylococcus aureus against ciprofloxacin oxidative damage. Fundam. Clin. Pharmacol. 24, 771-776. doi: 10.1111/j.1472-8206.2009. 00806.x

Palmer, A. C., and Kishony, R. (2013). Understanding, predicting and manipulating the genotypic evolution of antibiotic resistance. Nat. Rev. Genet. 14, 243-248. doi: $10.1038 / \mathrm{nrg} 3351$

Peter, B. J., Arsuaga, J., Breier, A. M., Khodursky, A. B., Brown, P. O., and Cozzarelli, N. R. (2004). Genomic transcriptional response to loss of chromosomal supercoiling in Escherichia coli. Genome Biol. 5:R87. doi: 10.1186/ gb-2004-5-11-r87

Rodríguez-Martínez, J. M., Cano, M. E., Velasco, C., Martínez-Martínez, L., and Pascual, Á (2011). Plasmid-mediated quinolone resistance: an update. J. Infect. Chemother. 17, 149-182. doi: 10.1007/s10156-010-0120-2

Rodríguez-Martínez, J. M., Fernández-Echauri, P., Fernández-Cuenca, F., Diaz de Alba, P., Briales, A., and Pascual, A. (2012). Genetic characterization of an extended-spectrum AmpC cephalosporinase with hydrolysing activity against fourth-generation cephalosporins in a clinical isolate of Enterobacter aerogenes selected in vivo. J. Antimicrob. Chemother. 67, 64-68. doi: 10.1093/jac/dkr423

Rodríguez-Martínez, J. M., Machuca, J., Cano, M. E., Calvo, J., MartínezMartínez, L., and Pascual, A. (2016a). Plasmid-mediated quinolone resistance: two decades on. Drug Resist. Updat. 29, 13-29. doi: 10.1016/j.drup.2016.09.001

Rodríguez-Martínez, J.-M., Santiso, R., Machuca, J., Bou, G., Pascual, Á, and Fernández, J. L. (2016b). Assessment of chromosomal DNA fragmentation by quinolones in an isogenic collection of Escherichia coli with defined resistance mechanisms. Microb. Drug Resist. 22, 354-359. doi: 10.1089/mdr.2015.0298

Seoane, A. S., and Levy, S. B. (1995). Characterization of MarR, the repressor of the multiple antibiotic resistance (mar) operon in Escherichia coli. J. Bacteriol. 177, 3414-3419. 
Shaw, K. J., Miller, N., Liu, X., Lerner, D., Wan, J., Bittner, A., et al. (2003). Comparison of the changes in global gene expression of Escherichia coli induced by four bactericidal agents. J. Mol. Microbiol. Biotechnol. 5, 105-122. doi: $10.1159 / 000069981$

Tatusov, R. L., Galperin, M. Y., Natale, D. A., and Koonin, E. V. (2000). The COG database: a tool for genome-scale analysis of protein functions and evolution. Nucleic Acids Res. 28, 33-36.

Thomas, V. C., Chittezham Thomas, V., Kinkead, L. C., Janssen, A., Schaeffer, C. R., Woods, K. M., et al. (2013). A dysfunctional tricarboxylic acid cycle enhances fitness of Staphylococcus epidermidis during $\beta$-lactam stress. MBio 4, e00437-13. doi: 10.1128/mBio.00437-13

Vila, J., Sáez-López, E., Johnson, J. R., Römling, U., Dobrindt, U., Cantón, R., et al. (2016). Escherichia coli: an old friend with new tidings. FEMS Microbiol. Rev. 40, 437-463. doi: 10.1093/femsre/fuw005

Wang, X., and Zhao, X. (2009). Contribution of oxidative damage to antimicrobial lethality. Antimicrob. Agents Chemother. 53, 1395-1402. doi: 10.1128/AAC. 01087-08

Webber, M. A., Ricci, V., Whitehead, R., Patel, M., Fookes, M., Ivens, A., et al. (2013). Clinically relevant mutant DNA gyrase alters supercoiling, changes the transcriptome, and confers multidrug resistance. MBio 4, e00273-13. doi: $10.1128 / \mathrm{mBio} .00273-13$
Yamaguchi, Y., and Inouye, M. (2011). Regulation of growth and death in Escherichia coli by toxin-antitoxin systems. Nat. Rev. Microbiol. 9, 779-790. doi: $10.1038 /$ nrmicro2651

Zhao, X., and Drlica, K. (2014). Reactive oxygen species and the bacterial response to lethal stress. Curr. Opin. Microbiol. 21, 1-6. doi: 10.1016/j.mib.2014. 06.008

Zhao, X., Hong, Y., and Drlica, K. (2015). Moving forward with reactive oxygen species involvement in antimicrobial lethality. J. Antimicrob. Chemother. 70, 639-642. doi: 10.1093/jac/dku463

Conflict of Interest Statement: The authors declare that the research was conducted in the absence of any commercial or financial relationships that could be construed as a potential conflict of interest.

Copyright (c) 2017 Machuca, Recacha, Briales, Díaz-de-Alba, Blazquez, Pascual and Rodríguez-Martinez. This is an open-access article distributed under the terms of the Creative Commons Attribution License (CC BY). The use, distribution or reproduction in other forums is permitted, provided the original author(s) or licensor are credited and that the original publication in this journal is cited, in accordance with accepted academic practice. No use, distribution or reproduction is permitted which does not comply with these terms. 